SIGraDi 2016, XX Congreso de la Sociedad Ibero-americana de Gráfica Digital

9-11, November, 2016 - Buenos Aires, Argentina

\title{
Trabajo conjunto inter/transdisciplinario para una propuesta concreta que dialoga con la sociedad y su medio. Citycrowdcreating
}

Cross-disciplinary work for a concrete proposal that interacts with society and the environment. Citycrowdcreating

\author{
Hernández, Silvia Patricia \\ FAUD. UNC Argentina \\ arqpatriciahernandez@gmail.com
}

Boccolini, Sara Maria

FAUD. UNC Argentina

saraboccolini@gmail.com

Chaves, Cristina

FAUD. UNC Argentina

cristinacha@hotmail.com
Genero, María Angeles

FAUD. UNC Argentina

mariangelagenero@gmail.com

Mari, Belén

FAUD. UNC Argentina

belma90@hotmail.com

Ron, Lucia

FAUD. UNC Argentina

lucia-ron@hotmail.com

\begin{abstract}
Working in pairs is collective collaboration. The collective intelligence, crowdthinking and its variations are part of today's language. Based on this concept, we propose to fill empty spaces from the current communication of culture from Goverment of Córdoba. There is an information movement and events in conection to crowd. We propose a device, that broadcasts the contents of the hashtag: \#quetenemoshoy, making it more popular, and in everyone's range. We set out a collaboration situation, with a concrete proposal, that includes dialogue with its surroundings. Articulating knowledge from different disciplines, between the goverment and the residents. Following neologism from Gutiérrez-Rubí y Freire (2013), we propose will be CITY-CROWD-CREATING.
\end{abstract}

Keywords: CrowdCreating; Microarchitecture; Inclusive; Communication.

\section{Introducción}

Siguiendo a Ramín (2014), los estudios de Ricci y Wiese (2011) han investigado la consolidación de un "imperativo de la colaboración" en el ámbito empresarial. Incluso, GutiérrezRubí y Freire (2013) hablan de "empresas crowd". Así como sus neologismos, crowdfunding, crowdthinking, crowdcuration, crowdcreating, coworking que muestran que la reciprocidad adquiere centralidad en los sectores más innovadores de la economía.

Por lo tanto, trabajar en pares es colaboración colectiva. Esas empresas forman plataformas de compartimiento de opiniones. Se autodefinen con objetivo de apoyar en la divulgación y dar oportunidad creciente de ideas, sean de producto material o inmaterial. Para que todo ocurra bien, aconsejan tener auto-organización en la persona que decide poner su idea para recibir comentarios de individuos participantes de sus plataformas. Son estos, integrantes de las redes sociales, la base del proceso de la denominación "crecimiento" o "multitud de ideas".
Esta base, es caracterizada por una amplia, diversa y de grupos pequeños. Estos participan en esas redes digitales, por eso se dice que la jerarquía de coordinación parte desde abajo. Entonces, la inteligencia colectiva, multitud de pensamientos, crowdthinking, y sus variaciones hacen parte del lenguaje actual. Sin embargo, no todos conocen sus reales valores.

Pensando en eso, la cuestión principal de esta investigación es buscar informaciones para llenar espacios vacíos de la comunicación actual en los sectores de ciencias sociales y humanas, específicamente, cultura y arquitectura. Existe movimiento de transmisión de datos y eventos relacionados a "crowd".

Así surge la hipótesis de proponer un espacio físico para retransmitir lo que difunde un hashtag. La propuesta en síntesis es construir un espacio real trabajando con lo digital.

Planteamos una situación de colaboración en varios aspectos, que sea concreta y que dialogue con su medio.

Es una propuesta de trabajo: 
- entre diversas profesiones, lo que le da el carácter interdisciplinario,

- entre el municipio y sus habitantes, o sea de servicio para la ciudad y sus diversos habitantes, con el objetivo de difundir e incluir

- de trabajo articulado con el gobierno de la ciudad, teniendo consideración especial a la difusión del hashtag \#quetenemoshoy, popularizándolo, llevándolo al alcance de todos.

$Y$ en este punto, siguiendo los neologismos de GutiérrezRubí y Freire (2013), sería CITY-CROWD-CREATING

\section{Gobierno municipal y sus objetivos de difusión}

El gobierno municipal de la ciudad de Córdoba, tiene varias áreas de trabajo. Está organizado por secretarías, que son las siguientes:

- Secretaría de Control y Fiscalización y Convivencia Ciudadana,

- Secretaría de Cultura, Secretaría de Economía y Finanzas,

- Secretaría de Educación

- Secretaría de Gobierno Participación Ciudadana y Desarrollo Social

Trabajan desde su portal, mejorando la Información y la Comunicación

(http://www2.cordoba.gov.ar/portal/index.php/secretaria-deeconomia-y-finanzas/subsec-de-tecnologias-de-informacion-ycomunicaciones/)

Ellos manifiestan que tiene el objetivo de actualizar y optimizar los sistemas de comunicaciones del Municipio y la red de conectividad del mismo para lograr un mejor funcionamiento de las diferentes áreas de la Municipalidad. Lo que les preocupa es que se vea directamente reflejado en la atención de los vecinos, para quienes trabajan.

Están convencidos de que con el fortalecimiento del área de Tecnologías de la Información y Comunicación en la Municipalidad de Córdoba, conseguirán más beneficios para los ciudadanos que se verán traducidos en una mejor atención a los cordobeses.

Trabajando con las TICs creen garantizar la actualización, la consolidación y el desarrollo de los sistemas informáticos municipales que permitan acercar al ciudadano herramientas más flexibles e integrales, permitiendo el acceso a la información municipal y garantizando la eficiencia y eficacia de los trámites.

Estos párrafos presentan los objetivos de la secretaría de comunicar por redes o virtualmente. La secretaría de cultura depende de la Municipalidad de Córdoba. Diseña y ejecuta políticas públicas que fomenten el desarrollo cultural de la ciudad de Córdoba. Tiene su sede en el cabildo de la ciudad, frente a la plaza mayor, en el centro de Córdoba. Su microsite es www.cultura.cordoba.gov.ar

Las manifestaciones culturales de la ciudad son amplias y variadas y esta secretaría se propone difundirlas. El teatro, la música, la plástica, el cine, las letras... todas las expresiones artísticas tienen su espacio de manifestación dentro de del ámbito municipal. En sus centros culturales y museos, los vecinos de la ciudad encuentran un lugar para disfrutar el arte, para producirlo, para compartirlo o rebatirlo. En el centro de la ciudad, en los barrios, en los espacios públicos, en los escenarios, siempre hay en Córdoba una oportunidad de producir e interpretar nuevos significados. Además se instruyen en talleres, cursos y conferencias de distintas disciplinas artísticas y recreativas.

Para difundir cultura de Córdoba tenemos los museos, Cineclub Municipal Hugo del Carril y la Feria del Libro Córdoba, la feria de Artesanos, la prestigiosa Orquesta de Cuerdas, el Coro Municipal, el Ensamble de Música Ciudadana además de la Noche de los muesos hasta el festival del choripán, muy variado.

Se dimensionó lo que hay que difundir considerando datos de la municipalidad, en el año 2015 hubieron hasta 10 actividades diarias, la mayoría gratis, en 28 espacios abiertos, contabilizándose un total de 1.500 .000 asistentes en el año 2015.

Combinados, sinérgicamente la dirección de comunicaciones con sus premisas tecnológicas y la Dirección de cultura con un amplio repertorio de actividades y su necesidad de difusión de las mismas es que se creó una dirección en twitter con el hashtag \#quetenemoshoy, (imagen 1).

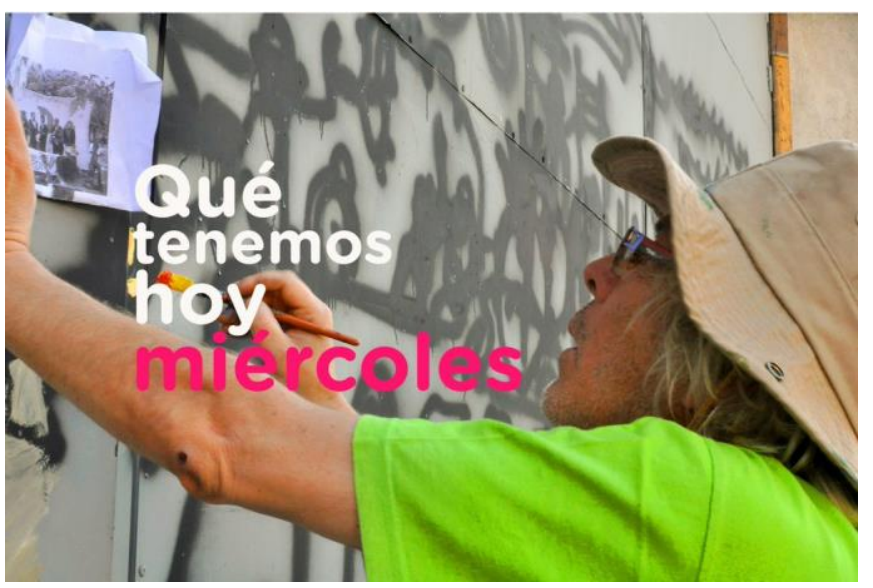

Imagen 1 - Cultura Cba @SecCulturaCba Jan 13

\#QuéTenemosHoy@ @AgendaGamba@turiscbaciudad http://htl.li/WZXvT 


\section{Dificultades}

Partiendo de la premisa de que Cultura es para esta secretaría una construcción colectiva de córdoba para Cordobeses es que hay que atender a la pluralidad de actores y usuarios.

Detectamos dos dificultades.

- La primera es que no todos tienen acceso o usan twitter.

Los que sí tienen acceso, no estamos seguros de que los lean. La publicidad, el marketing y la comunicación colapsan nuestro día a día y nuestro entorno (vallas, anuncios, campañas, banners, etc). Todos los anunciantes buscan llegar al máximo número de personas y, pese a la segmentación, son los números los que cuentan. Cada twitter, a las dos horas pasó a un historial difícil de reencontrar, llamado limbo twittero. Cada cuenta puede tener millones de seguidores pero no significa que leyeron todos y cada uno la información.

- La segunda es la necesidad manifestada por usuarios de esta ciudad de que alguien los asista cuando buscan la información.

Este equipo de investigación cuenta con encuestas realizadas por web en el año 2015. (http://encuestas2.unc.edu.ar/index.php/admin/statistics/sa/i ndex/surveyid/724792).

Basándonos en los resultados de la misma, podemos hacer las siguientes observaciones.

- Los usuarios se manifestaron en un $71 \%$ de acuerdo con que existan edificios públicos, de servicio o del gobierno que sean sustentables (ahorro de energía y disminución del impacto ambiental).

- En un $75,72 \%$ de los encuestados manifiesta que los edificios deben ser inclusivos y de bajo impacto ecológico.

Ante la pregunta Si usted se dirigiera a un edificio público y debiera anunciarse por recepción, la opción que usted consideraría como más positiva sería:

1. Prefiero resolver mis consultas yo mismo en un puesto de autoconsulta (A1) ......................28-19.58\%

2. Prefiero que me asistan mientras realizo

operaciones en un puesto de autoconsulta (A2) 52- 36.36\%

3. Prefiero ser atendido por un empleado (A3) 63- $44.06 \%$

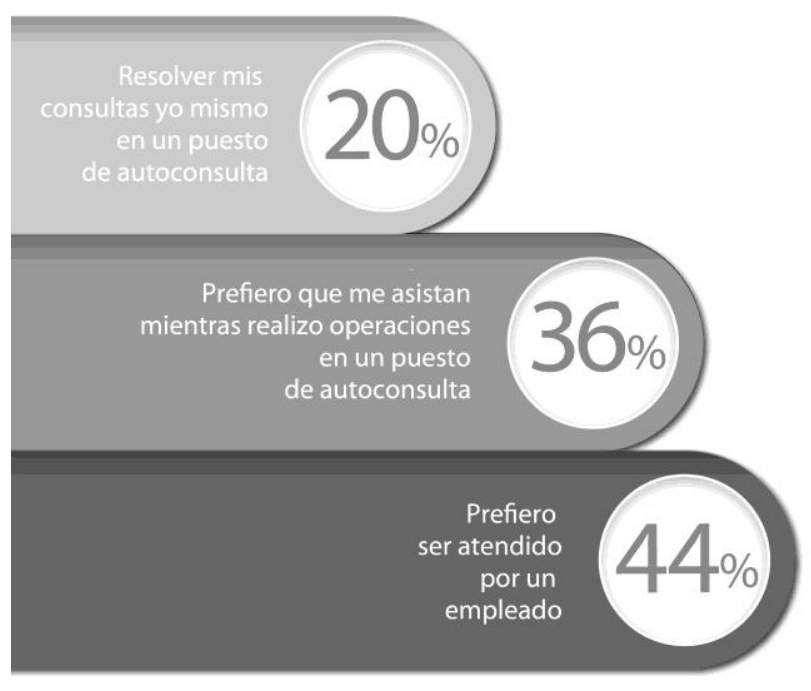

Imagen $\mathrm{n}^{\circ} 2$, gráfico encuestas, imagen propia.

Sorprendentemente sólo el $28 \%$ de los encuestados manifestó querer resolver por sí mismo sus consultas, siendo un universo donde el 93,7 \% posee internet en su hogar. Resta un 63\% prefiere ser atendido por un empleado.(imagen 2).

\section{Propuesta}

Es en esta confirmación de dato que nos animamos a proponer un trabajo conjunto para hacer visible y posible para muchos las actividades de la secretaría de cultura. Considerando que no todas las personas tienen acceso al twitter y que la cultura es un derecho humano. Acercamos la posibilidad de vivir el espacio público con arte y deslumbrarse con los artistas.

Proponemos un espacio donde se puedan consultar las propuestas culturales y además poder imprimir un ticket de entrada, o con la información de la actividad. Estos servicios también sinérgicamente reforzarán el hashtag, o sea la información via redes. Así estaremos trabajando en este CITY-CROWCREATING

\section{Sitio- Espacios residuales, Instersticios espaciales}

Elegimos un sitio neurálgico de las actividades culturales, en la media legua de oro, La Media Legua de Oro Cultural es un recorrido que abarca cerca de 2.500 metros entre la histórica Plaza San Martín, parte del teatro Real, hacia el barrio de Nueva Córdoba, descansando sobre el majestuoso Parque Sarmiento, diseñado hace más de un siglo por el Arq. Carlos Thays.( Imagen 3) 


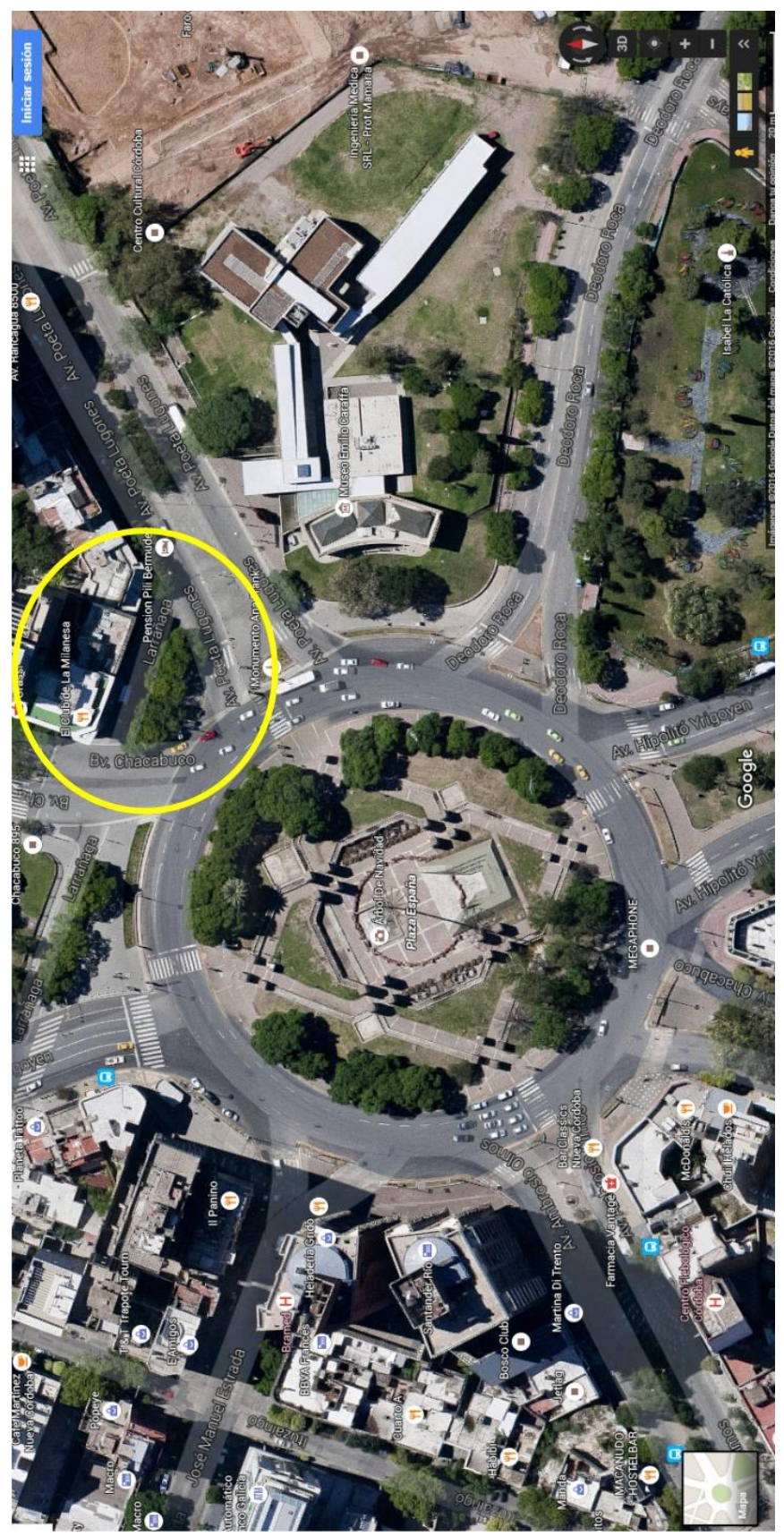

Imagen n³, aproximación al sitio, imagen google maps

La plaza España es un punto vial importante ya que allí se interseccionan seis avenidas, generando ocho salidas a cada una de ellas. Los museos de la zona, son Museo Superior de Bellas Artes Evita (ex palacio Ferreyra), museo Caraffa, Museo Palacio Dionisi y el Archivo Histórico Provincial con su polular faro.
Este cruce de avenidas con la rotonda generó espacios intersticiales aptos para el trabajo. Además al estar ubicados con el barrio Nueva Córdoba, que es un barrio planificado a fines del Siglo XIX en conjunto con el Parque Sarmiento por el arquitecto y urbanista Carlos Thays. En sus principios fue un barrio de clase alta con lujosas residencias. En la segunda mitad del siglo tuvo un rápido cambio hacia una zona de edificaciones en altura, poblada sobre todo por estudiantes de la cercana Universidad Nacional de Córdoba. Tiene una población de 37.000 habitantes, (censo 2010) y actualmente es el barrio de la zona céntrica con mayor densidad poblacional.

Reconocemos en la compleja trama urbana de las ciudades espacios llamados residuales que en realidad son instersticios espaciales. Considerando como el espacio que media entre dos partes de distintos objetos o entre dos del mismo cuerpo.

En la tarea de aproximarse a nuevas lecturas de territorios, y en este crowcreating, se trabaja a partir de investigar los procesos actuales que intervienen en la espacialidad intersticial en términos de reinterpretación como potencial de nuevas propuestas, con el objetivo de trabajar en un intersticio comunicacional que hemos detectado en el área de Cultura municipal.

La propuesta tendrá nuevas configuraciones a modo de adaptación, o engrosamiento o completamiento de estos intersticios. Este CityCrowCreating, enfoca a trabajar en espacios intersticiales de la ciudad para la ciudad y sus habitantes difundiendo las manifestaciones culturales que se dan en Córdoba.( imagen 4 y 5)

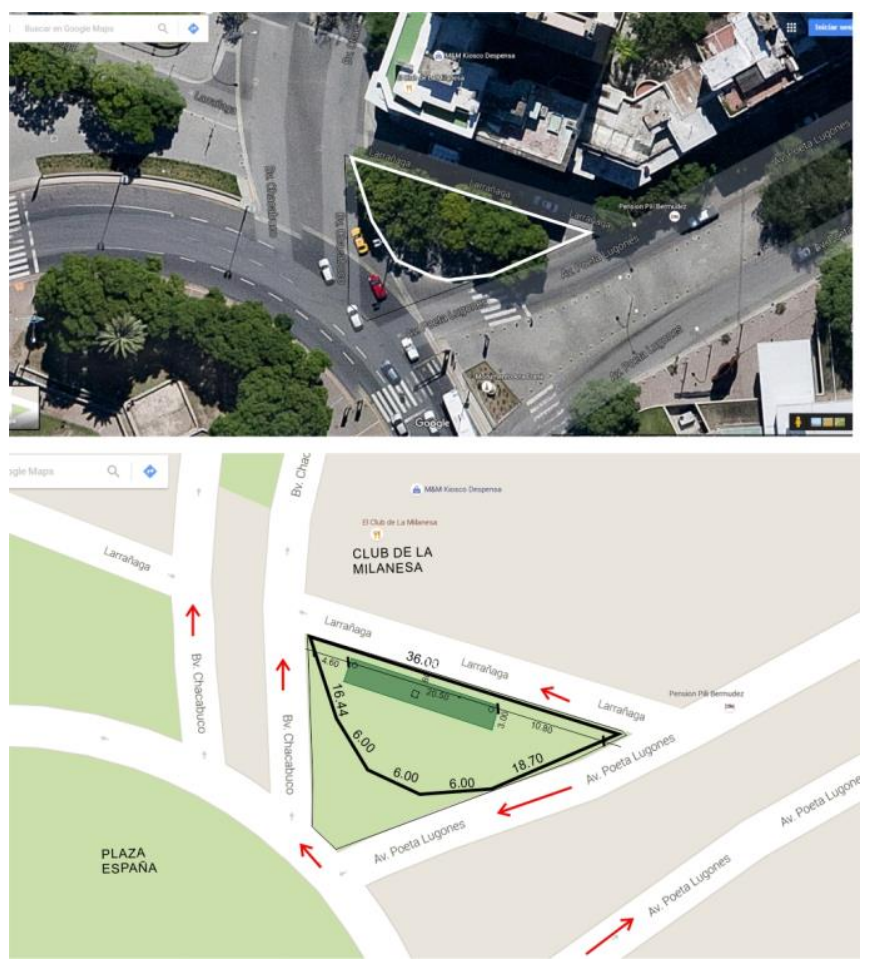




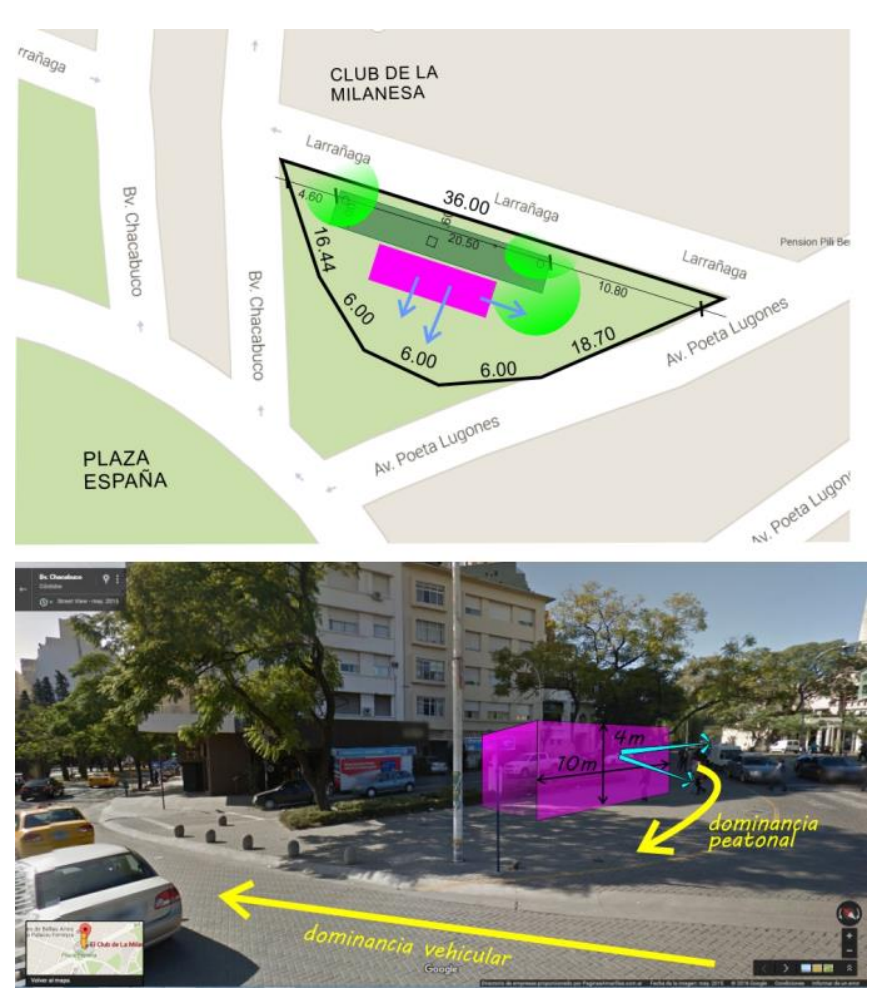

Imagen $\mathrm{n}^{\circ} \mathbf{4}$, sitio a intervenir, imagen propia. Imagen $n^{\circ} 5$, área de posibible intervención, imagen propia.

\section{Diseño del Dispositivo}

Se proyecta el diseño y desarrollo de una micro-arquitectura que actúe de punto físico y visual de la plataforma virtual (hoy existente) de la Municipalidad de Córdoba. La característica principal del \#quetenemoshoy y nuestro disparador de diseño, que es una plataforma de divulgación digital nuclea a todas las actividades de índole cultural que se desarrollan por día en la ciudad, una especie de agenda. Se promueve así la divulgación y generación de oportunidades crecientes a ideas, grupos, espacios de la ciudad. El dispositivo será soporte secundario de las actividades que realiza la municipalidad en el lugar (por ej., el arbolito de navidad y encender las luces/evento no del gusto de todos, pero con convocatoria, o actividades del parque Sarmiento: el festival del choripán, 21 de septiembre, día de la primavera, día del niño, corsos de carnaval, etc.

El portal funciona como un dispositivo atractor de las actividades culturales que organiza la municipalidad. Se coloca en un nodo central de la red cultural espacial de la ciudad (la red formada por el centro histórico, la media legua de oro, ciudad universitaria, Nueva Córdoba y Güemes y La Cañada), para captar el flujo de visitantes y vecinos que pasean por la zona y atraerlos a actividades que se realizan en las cercanías o en otras partes de la ciudad.

Funciona como un portal que motiva la búsqueda de eventos que ocurren en nodos secundarios de la red, e invita a descubrir la complejidad y riqueza de los eventos culturales de la ciudad.

El \#quetenemoshoy se inserta en medio del recorrido cultural hegemónico y estimula la curiosidad y exploración de otras partes de la ciudad, de actividades novedosas, de nuevos escenarios y otros actores. La construcción y fortalecimiento de redes urbano-culturales alternativas en el espacio urbano se realiza a partir de plantear, esbozar, proponer, futuras conexiones en el espacio público en este dispositivo, que funciona como un verdadero portal a nuevas experiencias en la ciudad.

\section{Funciones del dispositivo \#quétenemoshoy}

Cómo llevar esa agenda cultural a una cartelera real? Se trabajará con la virtualidad, con imágenes en vivo, pantallas e imágenes 2D.

Se ha considerado incluir los siguientes lineamientos en el proyecto. Responderá a ese canal puente peatonal que une el museo Evita y el Caraffa, dentro de la Media Legua de Oro.

Permitirá explorar actividades pasadas (tener en cuenta que varias personas deben poder hacerlo simultáneamente, así se propone el uso de gadgets que no estén ocupando el espacio principal); actividades presentes (incluso, con pantallas donde los usuarios pueden permanecer por unos minutos viendo el espectáculo -me refiero a los gratuitos, como las performance del coro municipal, etc.. Estas actividades además de difusión, resultan un llamador para que la gente vaya al lugar donde se está produciendo el evento. (imagen 6)

Además anuncia eventos que vienen, se exponen actividades próximas, para servir de invitación efectiva a esos eventos.

El dispositivo tendrá partes móviles/plegables/o tipo "sacar y poner" que se puedan instalar según la ocasión. Se fragmentará para vincularse a las actividades cercanas

En cuanto a su misión crowd, se propone expandir la red de escenarios culturales y nodos de interacción con visitantes y vecinos.

El dispositivo incluirá acceso al portal de transporte público, para poder conocer cómo llegar a las actividades propuestas, y punto de venta y carga de la RedBus.(imagen7) 

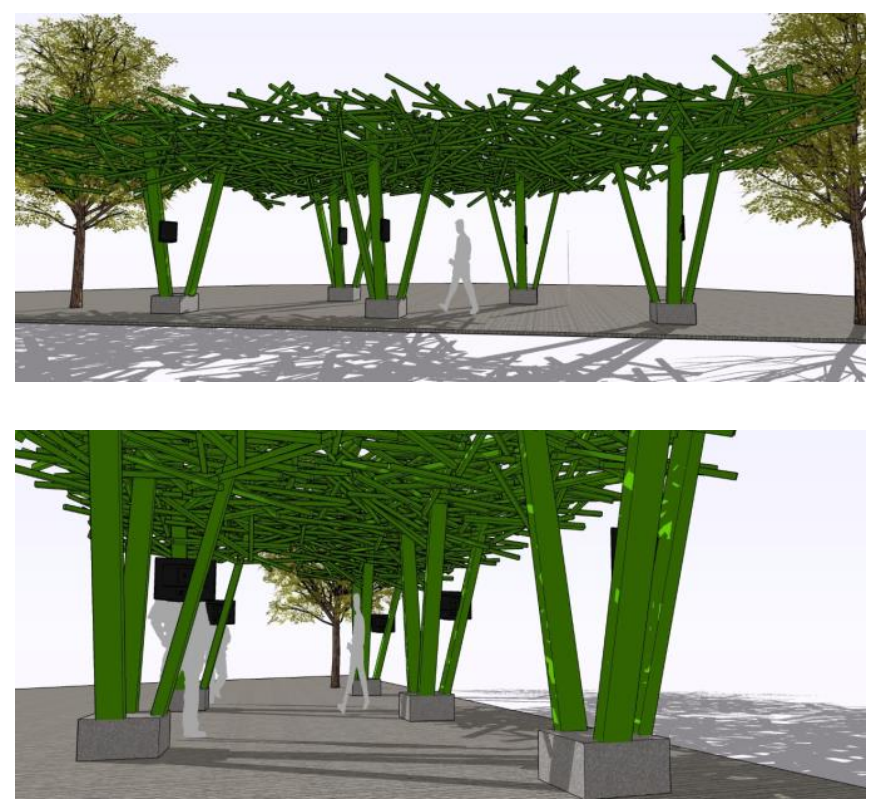

Imagen $\mathrm{n}^{\circ} 6$ y 7 , primera intervención y propuesta, imagen propia.

\section{Conclusiones y Discusión}

El tema resulta actual y favorecedor para confronto de pensamientos y líneas de investigación. Advierte y sugiere la necesidad de adaptaciones para las diferentes escalas de la enseñanza, del trabajo conjunto, para la ciudad. Poder trabajar en una línea de CityCrowdCreating, motiva y organiza esta investigación, marcando nuevas líneas de trabajo y objetivos del proyecto, enriqueciéndolo.

En cuanto a su misión crowd, se propone expandir la red de escenarios culturales y nodos de interacción con visitantes y vecinos. Proponemos en una segunda etapa, en donde se trabajará con encuestas a los habitantes de Córdoba para que participen en la definición del proyecto, opinen y definan requerimientos específicos. Además nos marca una instancia de apertura también en los contenidos de la difusión cultural. En qué medida están participando los hacedores culturales, verdaderos protagonistas? Cómo prefieren ellos que se difundan sus propuestas?

Nos queda un gran desafío que es poder desarrollar el legajo e implementarlo. Estamos convencidos que la arquitectura surge de una forma de conocimiento compartido que va más allá del objeto arquitectónico, para lo cual esta línea de trabajo e investigación redundará en beneficios para la ciudad y sus habitantes, trabajando con la inclusividad y la sustentabilidad.

\section{Agradecimientos}

Agradecemos a los asesores Ingenieros, Lucio Fernando Madussi, (lucholemm@gmail.com); Hugo

Dallegre(hdallegre@gmail.com); y al ing marcos

Blasco,(marcos.ielect@gmail.com) de la facultad de CEFyN UNC.

\section{Referencias}

Alessi, C. (2014). Dopo gli anni Zero: il nuovo design italiano. Roma, Editori Laterza.

Bauwens, M. (2005). "The Political Economy of Peer Production", Pos-Autisc Economics Review 37, [en línea] [site], Consulta: fecha

Drucker, P.F. (1968). The age of discontinuity: guidelineas to our changing, Nueva York, Harper \& Row Society.

Gutiérrez-Rubí, A. y J. FREIRE, eds. (2013). Manifesto Crowd, Laboratorio de tendencias, Kindle e-book.

Masuda, Y. (1984). La sociedad informatizada como sociedad postindustrial, Madrid, Fundesco-Tecnos.

Portal do Ministério da Educação - MEC (2016). Certificado brasileiro CERTIFIC do governo do Ministério da Educação. Disponible en: $<$ http://portal.mec.gov.br/index.php?option=com_content\&view=articl e\&id=15266\&ltemid=800 . . Acceso en 26 de marzo de 2016.

Obrist, H. U. (2010). Tomás Maldonado: Arte e artefatti - Intervista di Hans Ulrich Obrist. Milano, Feltrinelli.

Ramís, A. (2014). Los bienes comunes intangibles en el capitalismo cognitivo. Recerca, revista de Pensamento I Análisi, N.15, pp. 109129.

Touraine, A. (1969) La sociedad pos-industrial, Barcelona, Ariel [Traducción de Juan -Ramón Capella y Francisco J. Fernández Buey]

http://www2.cordoba.gov.ar/portal/ recuperado 19 de junio 2016

https://twitter.com/hashtag/quetenemoshoy?src=hash, recuperado 19 de junio 2016

CORDOBA UNA CIUDAD EN CIFRAS, www2.cordoba.gov.ar/portal/wpcontent/uploads/downloads/2012/10/Cordoba-una-Ciudad-en-Cifras2012.pdf 